\title{
Comparison of Clinician Suspicion Versus a Clinical Prediction Rule in Identifying Children at Risk for Intra-abdominal Injuries After Blunt Torso Trauma
}

Prashant Mahajan, MD, MPH, MBA, Nathan Kuppermann, MD, MPH, Michael Tunik, MD, Kenneth Yen, MD, MS, Shireen M. Atabaki, MD, MPH, Lois K. Lee, MD, MPH, Angela M. Ellison, MD, MSc, Bema K. Bonsu, MD, Cody S. Olsen, MS, Larry Cook, PhD, Maria Y. Kwok, MD, MPH, Kathleen Lillis, MD, James F. Holmes, MD, MPH, for the Intra-abdominal Injury Study Group of the Pediatric Emergency Care Applied Research Network (PECARN)

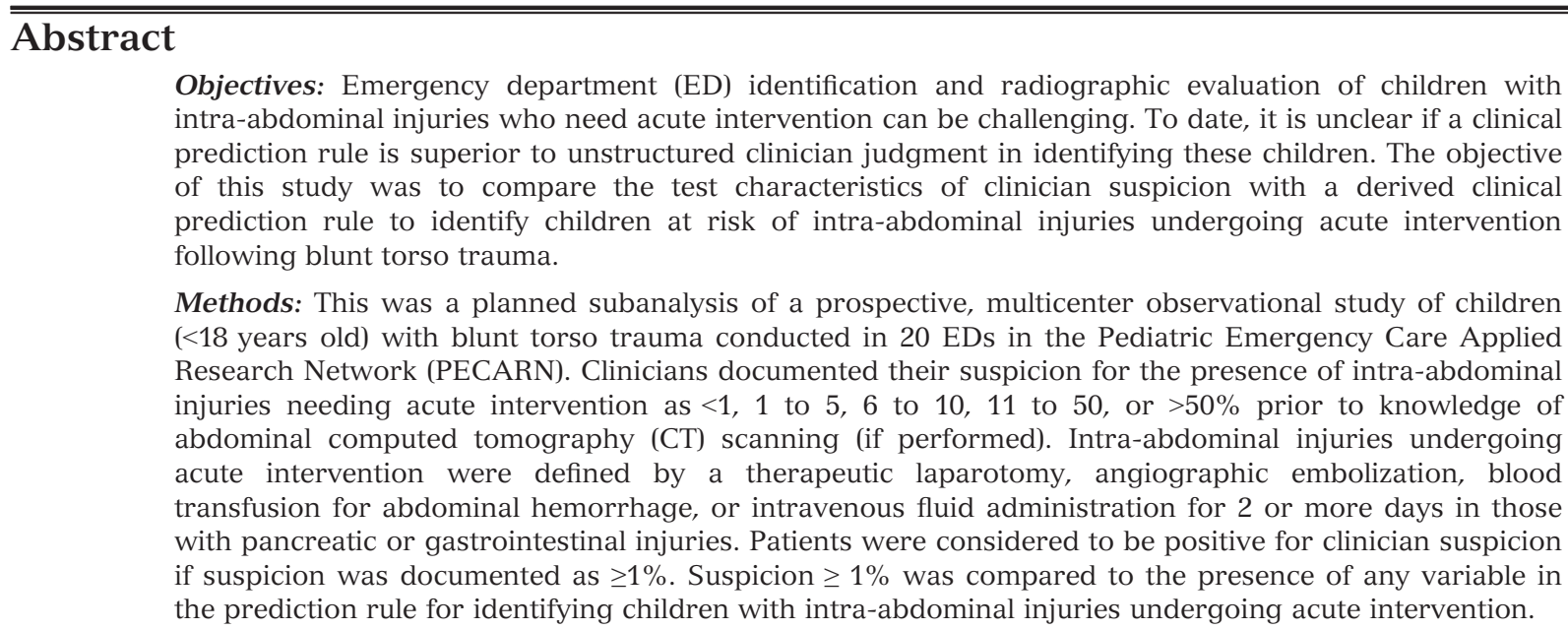

From the Division of Emergency Medicine, Department of Pediatrics, Children's Hospital of Michigan, Wayne State University (PM), Detroit, MI; the Departments of Emergency Medicine (NK, JFH) and Pediatrics (NK), University of California, Davis, Sacramento, CA; the Departments of Emergency Medicine and Pediatrics, New York University School of Medicine, Bellevue Hospital Center (MT), New York, NY; the Department of Pediatrics, Section of Emergency Medicine, Medical College of Wisconsin (KY), Milwaukee, WI; the Division of Pediatric Emergency Medicine, Children's National Medical Center (SMA), Washington, DC; the Division of Emergency Medicine, Boston Children's Hospital, Harvard Medical School (LKL), Boston, MA; the Department of Emergency Medicine, Children's Hospital of Philadelphia (AE), Philadelphia, PA; the Division of Emergency Medicine, Department of Pediatrics, Nationwide Children's Hospital (BKB), Columbus, OH; the Department of Pediatrics, University of Utah School of Medicine (CSO, LC), Salt Lake City, UT; the Department of Pediatrics, New York Presbyterian-Morgan Stanley Children's Hospital, Columbia University Medical Center (MYK), New York, NY; and Pediatric Emergency Medicine, Women and Children's Hospital of Buffalo (KL), Buffalo, NY.

Received December 22, 2014; revision received March 25, 2015; accepted April 24, 2015.

Presented in part at the American College of Emergency Physicians Research Forum, Denver, CO, October 2012.

This work was supported by a grant from the Centers for Disease Control and Prevention 1 R49CE00100201.

PECARN is supported by the Health Resources and Services Administration (HRSA), Maternal and Child Health Bureau (MCHB), Emergency Medical Services for Children (EMSC) Program through the following cooperative agreements: U03MC00001, U03MC00003, U03MC00006, U03MC00007, U03MC00008, U03MC22684, and U03MC22685.

Dr. Bonsu, an associate editor at this journal, had no role in the peer-review process or publication decision for this paper.

The authors have no potential conflicts to disclose.

Supervising Editor: Jennifer Walthall, MD, MPH.

Address for correspondence and reprints: Prashant Mahajan, MD, MPH, MBA; e-mail: pmahajan@dmc.org. 
Results: Clinicians recorded their suspicion in 11,919 (99\%) of 12,044 patients enrolled in the parent study. Intra-abdominal injuries undergoing acute intervention were diagnosed in $203(2 \%)$ patients. Abdominal CT scans were obtained in the ED in 2,302 of the 2,667 (86\%, 95\% confidence interval $[\mathrm{CI}]=85 \%$ to $88 \%)$ enrolled patients with clinician suspicion $\geq 1 \%$ and in 3,016 of the $9,252(33 \%, 95 \%$ $\mathrm{CI}=32 \%$ to $34 \%$ ) patients with clinician suspicion $<1 \%$. Sensitivity of the prediction rule for intraabdominal injuries undergoing acute intervention (197 of 203; 97.0\%, 95\% CI $=93.7 \%$ to $98.9 \%$ ) was higher than that of clinician suspicion $\geq 1 \%$ (168 of $203 ; 82.8 \%, 95 \%$ CI $=76.9 \%$ to $87.7 \%$; difference $=$ $14.2 \%, 95 \% \mathrm{CI}=8.6 \%$ to $20.0 \%)$. Specificity of the prediction rule $(4,979$ of the 11,$716 ; 42.5 \%, 95 \%$ $\mathrm{CI}=41.6 \%$ to $43.4 \%)$, however, was lower than that of clinician suspicion $(9,217$ of the $11,716,78.7 \%$, $95 \% \mathrm{CI}=77.9 \%$ to $79.4 \%$; difference $=-36.2 \%, 95 \% \mathrm{CI}=-37.3 \%$ to $-35.0 \%)$. Thirty-five $(0.4 \%, 95 \%$ $\mathrm{CI}=0.3 \%$ to $0.5 \%$ ) patients with clinician suspicion $<1 \%$ had intra-abdominal injuries that underwent acute intervention.

Conclusions: The derived clinical prediction rule had a significantly higher sensitivity, but lower specificity, than clinician suspicion for identifying children with intra-abdominal injuries undergoing acute intervention. The higher specificity of clinician suspicion, however, did not translate into clinical practice, as clinicians frequently obtained abdominal CT scans in patients they considered very low risk. If validated, this prediction rule can assist in clinical decision-making around abdominal CT use in children with blunt torso trauma.

ACADEMIC EMERGENCY MEDICINE 2015;22:1034-1041 (c) 2015 by the Society for Academic Emergency Medicine

$\mathrm{T}$ rauma is the leading cause of death in children, with blunt torso trauma contributing substantially. ${ }^{1}$ Abdominal computed tomography (CT) is used with increasing frequency as it is the reference standard for detecting intra-abdominal injuries..$^{2-4}$ Routine CT scanning of the abdomen, however, has significant drawbacks, the most concerning of which is exposure to high doses of ionizing radiation. This is particularly worrisome in children because of their higher risk for developing radiation-induced malignancies. ${ }^{5-10}$

CT scanning of trauma patients has increased substantially in recent decades despite limited evidence supporting its increased use. ${ }^{11}$ Furthermore, there is substantial variation among clinicians and centers in the use of abdominal CT for injured children. ${ }^{3,11}$ Evidence suggests that clinician suspicion is not highly accurate and clinicians frequently order advanced imaging despite very low suspicion for clinically important injuries. ${ }^{12-15}$ Evidence-based clinical prediction rules, however, can assist clinical decision-making and reduce unnecessary variation. ${ }^{16,17}$ A clinical prediction rule to identify children at very low risk for intra-abdominal injuries undergoing acute intervention was recently derived. ${ }^{18}$ It is unclear, however, how the derived clinical prediction rule compares to unstructured clinician suspicion for identifying children with these injuries.

In this study, we sought to compare the test characteristics of clinician suspicion with a derived clinical prediction rule to identify children at very low risk of intra-abdominal injuries undergoing acute intervention. We also sought to determine the rationale for CT use when clinician suspicion was very low $(<1 \%)$. We hypothesized that a derived clinical prediction rule would have a higher sensitivity than clinician suspicion.

\section{METHODS}

\section{Study Design}

This was a planned secondary analysis of a prospective observational cohort study of children with blunt torso trauma conducted at 20 emergency departments (EDs) within the Pediatric Emergency Care Applied Research Network (PECARN). Institutional review boards at all participating sites approved this study.

\section{Study Setting and Population}

Study patients were enrolled from May 2007 to January 2010. The parent study included children younger than 18 years old with blunt torso trauma evaluated at participating PECARN EDs. Patients were excluded for any of the following: injury occurring $>24$ hours prior to presentation, penetrating trauma, preexisting neurologic disorders preventing reliable examination, known pregnancy, or transfer from another hospital with prior abdominal CT scanning or diagnostic peritoneal lavage. For this analysis we additionally excluded those patients for whom the clinician did not document his or her clinical suspicion of intra-abdominal injury undergoing acute intervention on the data collection form.

\section{Study Protocol}

The methods for the parent study have been previously described. $^{18}$ Clinicians (faculty physicians, fellows, nurse practitioners, or physician assistants) completed standardized data collection forms prior to abdominal CT (if performed). Faculty or fellow physicians verified the information if the form was completed by a nurse practitioner or physician assistant. Abdominal CT scans were obtained based on the decisions of the physicians providing care in the ED. For those undergoing abdominal CT scans, clinicians recorded the clinical variables influencing their decisions and prospectively recorded their suspicions of intra-abdominal injuries undergoing acute intervention on an ordinal scale with values of $<1,1$ to 5,6 to 10,11 to 50 , or $>50 \%$. Clinician suspicion was documented in all patients (irrespective of the performance of an abdominal CT) and prior to awareness of abdominal CT results if such imaging was performed. At the time of patient enrollment, clinicians were unaware of the 
Table 1

Clinical Prediction Rule Derived for Identifying Children at Very Low Risk for Intra-abdominal Injury Undergoing Acute Intervention $^{18}$

No evidence of abdominal wall trauma or seat belt sign Glasgow Coma Scale score $>13$

No abdominal tenderness

No evidence of thoracic wall trauma

No complaints of abdominal pain

No decreased breath sounds

No history of vomiting after the injury

specific variables in the clinical prediction rule, as the rule was not yet derived.

\section{Outcome Measures}

Intra-abdominal injury was defined as any injury identified to the following intra-abdominal structures: spleen, liver, urinary tract (kidney to the urinary bladder), gastrointestinal tract (from the stomach to the sigmoid colon including the mesentery), pancreas, gallbladder, adrenal gland, intra-abdominal vascular structure, or traumatic fascial defect. Intra-abdominal injury undergoing acute intervention was defined by death due to the abdominal injury, surgical intervention at laparotomy, angiographic embolization due to bleeding from the injury, blood transfusion for anemia secondary to intra-abdominal hemorrhage from the injury, or administration of intravenous fluids for at least two nights in those patients with pancreatic or gastrointestinal injuries.

To comprehensively identify children with intra-abdominal injuries undergoing acute intervention, we performed clinical follow-up on all patients. We reviewed medical records of all admitted patients and conducted a telephone follow-up survey at least 1 week after the index ED evaluation for those discharged from the ED. If telephone follow-up was unsuccessful, the same follow-up survey was mailed. If this was not returned, we reviewed medical records, ED process improvement records, local trauma registries, and morgue records to identify any potentially missed patients with intra-abdominal injuries.

\section{Data Analysis}

We summarized data using descriptive statistics with 95\% confidence intervals (CIs). Clinician suspicion of intra-abdominal injury undergoing acute intervention was considered positive if the clinician gauged the risk of this outcome to be $\geq 1 \%$. The clinician suspicion was considered to be negative (i.e., patient at very low risk) if the risk documented was $<1 \%$. Patients were considered positive for the clinical prediction rule if they had any one of the variables in the prediction rule (Table 1) documented as present. $^{18}$ In contrast, patients were considered to be negative (i.e., at very low risk) for the prediction rule if they did not exhibit any of the variables of the prediction rule.

We calculated the sensitivity, specificity, negative predictive value (NPV), and positive predictive values (PPV) of clinician suspicion of intra-abdominal injury undergoing acute intervention, along with positive likelihood ratio $(\mathrm{LR}+$ ) and negative likelihood ratio (LR-). We also calculated the same test characteristics for the derived clinical prediction rule. We then compared the test performance of clinician suspicion to the categorization of risk by the derived prediction rule and report rate differences with 95\% CIs. Sample size for this data set was calculated based on deriving a clinical prediction rule. ${ }^{18}$ There was no sample size calculation for this specific analysis. All statistical analyses were performed using SAS 9.3 software.

\section{RESULTS}

There were $12,044(81 \%)$ of 14,882 eligible patients enrolled in the parent study. From this cohort, 11,919 (99\%) patients met inclusion criteria and constituted the study population. The median age of the study cohort was 11 years (range $=2$ days to 17.9 years; interquartile range $=5.8$ to 15.0 years), and 7,308 (61\%) were male. Intra-abdominal injuries undergoing acute intervention occurred in $203(1.7 \%, 95 \%$ CI $=1.5 \%$ to $2.0 \%)$ patients.

The rates of abdominal CT scanning and intraabdominal injuries undergoing acute intervention increased as the clinicians' suspicions increased (Table 2). Thirty-five $(0.4 \%, 95 \% \mathrm{CI}=0.3 \%$ to $0.5 \%$ ) patients with clinician suspicion documented as $<1 \%$

Table 2

Clinician Suspicion for Intra-abdominal Injury Undergoing Acute Intervention and Abdominal CT Rates*

\begin{tabular}{|c|c|c|c|}
\hline Clinician Suspicion & Number Enrolled & Abdominal CT & $\begin{array}{c}\text { Intra-abdominal Injuries Undergoing } \\
\text { Acute Intervention }\end{array}$ \\
\hline$<1 \%$ & 9,252 & 3,016 (32.6) 31.6-33.6 & $35(0.4) 0.3-0.5$ \\
\hline $1 \%-5 \%$ & 1,793 & $1,484(82.8) 80.9-84.5$ & $40(2.2) 1.6-3.0$ \\
\hline $6 \%-10 \%$ & 506 & 476 (94.1) 91.6-96.0 & $33(6.5)$ 4.5-9.0 \\
\hline $11 \%-50 \%$ & 281 & 269 (95.7) 92.7-97.8 & 59 (21.0) 16.4-26.2 \\
\hline$>50 \%$ & $87 *$ & 73 (83.9) 74.5-90.9 & 36 (41.4) 30.9-52.5 \\
\hline Totals & 11,919 & 5,318 & 203 \\
\hline \multicolumn{4}{|c|}{$\begin{array}{l}\text { Data are reported as } n(\%) 95 \% \mathrm{Cl} \text {. } \\
\text { *Fourteen patients with clinician suspicion }>50 \% \text { did not undergo abdominal CT. These patients had the following: six patients } \\
\text { died without abdominal imaging, six patients underwent laparotomy, one patient had laparoscopy, and one patient had an } \\
\text { abdominal ultrasound and was admitted for observation. } \\
\text { CT = computed tomography. }\end{array}$} \\
\hline
\end{tabular}


had intra-abdominal injuries undergoing acute intervention. Three $(9 \%)$ of these 35 patients also were very low risk by the clinical prediction rule. Figure 1 shows the proportion of patients with intra-abdominal injuries for whom clinician suspicion was documented; it also shows the proportion with such injuries for whom clinician suspicion was $<1 \%$. Six patients with intraabdominal injuries undergoing acute intervention were considered very low risk by the clinical prediction rule. The clinician suspicions in these six patients were as follows: three were $<1 \%$, two were $1 \%$ to $5 \%$, and one was $6 \%$ to $10 \%$. The three patients undergoing therapy for their intra-abdominal injuries but not identified by the clinical prediction rule and given $<1 \%$ risk by clinical suspicion were as follows: a 2 year-old struck by a car and found to have gross hematuria and a renal injury, a 2 year-old with nonaccidental trauma found to have liver and gastrointestinal injuries, and a 17-yearold ejected after a motor vehicle collision found to have a splenic injury.

The test characteristics of clinician suspicion and the prediction rule are presented in Table 3 . The derived clinical prediction rule was more sensitive than clinician suspicion, but was less specific. Therapies for patients with intra-abdominal injuries with clinician suspicions $\geq$ $1 \%$ and $<1 \%$ are presented in Table 4 .

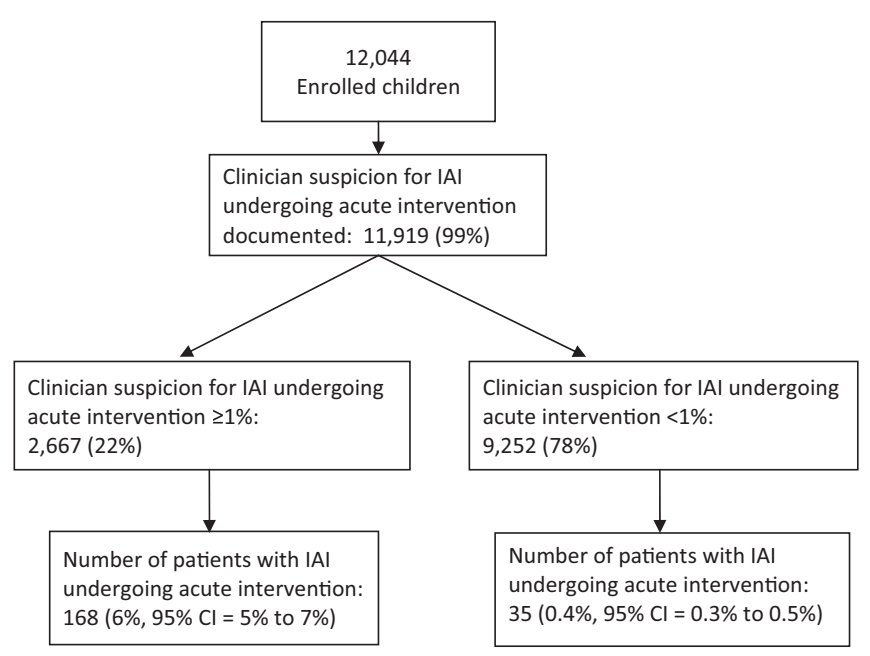

Figure 1. Patient flow chart. $|A|=$ intra-abdominal injury.
Abdominal CT scans were obtained in the ED for $2,302(86 \%, 95 \% \quad \mathrm{CI}=85 \%$ to $88 \%)$ of the 2,667 patients with clinician suspicion $\geq 1 \%$. Clinicians, however, frequently did not practice in accordance with their reported clinical suspicions, as CT scans were obtained in $3,016(33 \%, 95 \%$ CI $=32 \%$ to $34 \%)$ of 9,252 patients considered at very low clinician suspicion $(<1 \%)$. Table 5 lists the indications that most influenced the clinicians' decisions to order abdominal CT scans and the rates of intra-abdominal injury undergoing therapeutic intervention and laparotomy when the clinicians' suspicions were $<1 \%$.

\section{DISCUSSION}

In this subanalysis of a large multicenter study in which we enrolled a diverse population of children with blunt torso trauma, we demonstrated that a derived clinical prediction rule has substantially higher sensitivity than unstructured clinician suspicion in identifying children with intra-abdominal injuries undergoing acute intervention. Clinician suspicion, however, had a higher specificity. Despite the ability to correctly identify children who did not have intra-abdominal injuries undergoing acute intervention (i.e., higher specificity of clinician suspicion than the clinical prediction rule), clinicians nevertheless obtained abdominal CT scans in one-third of children they classified as having $<1 \%$ risk. This suggests an opportunity exists to reduce use of unnecessary abdominal CT scans in children by appropriate use of a clinical prediction rule.

The diagnostic evaluation of children with blunt abdominal trauma may be difficult due to uncertainties in the patient history and physical examination. Because of this uncertainty, and the lack of definitive research regarding specific indications for abdominal CT use, clinicians obtain abdominal CT scans in a large number of children with blunt trauma, including many who do not have intra-abdominal injuries. ${ }^{19}$ Consistent with prior studies, the parent study of the current analysis ${ }^{18}$ revealed the prevalence rate of intra-abdominal injury undergoing acute intervention to be less than $2 \%$ of children with blunt torso trauma, while nearly half of the children presenting with blunt torso trauma underwent abdominal CT scaning. ${ }^{15,20-22}$

In both adult and pediatric trauma patients, CT use has rapidly increased in the ED for many conditions. $3,12,23,24$

Table 3

Comparison of Test Characteristics Between Clinician Suspicion and the Prediction Rule for Intra-abdominal Injury Undergoing Acute Intervention

\begin{tabular}{|c|c|c|c|}
\hline Characteristic & Prediction Rule & Clinician Suspicion $\geq 1 \%$ & Difference \\
\hline Sensitivity & 97.0 (93.7 to 98.6$)$ & 82.8 (77.0 to 87.3$)$ & 14 (8 to 20$)$ \\
\hline Specificity & 42.5 (41.6 to 43.4$)$ & 78.7 (77.9 to 79.4$)$ & $-36(-37$ to -35$)$ \\
\hline NPV & 99.9 (99.7 to 99.9$)$ & 99.6 (99.5 to 99.7 ) & $0.3(0.1$ to 0.4$)$ \\
\hline PPV & $2.8(2.5$ to 3.3$)$ & $6.3(5.4$ to 7.3$)$ & $-3.5(-4.5$ to -2.5 \\
\hline LR+ & 1.7 (1.6 to 1.8$)$ & 3.9 (3.6 to 4.2$)$ & \\
\hline LR- & $0.1(0.0$ to 0.2$)$ & $0.2(0.2$ to 0.3$)$ & \\
\hline
\end{tabular}


Table 4

Types of Intervention Based on Clinician Suspicion of Intra-abdominal Injury Undergoing Acute Intervention ( $<1 \%$ and $\geq 1 \%)$

\begin{tabular}{|c|c|c|}
\hline \multirow[b]{2}{*}{ Type of Intervention* } & \multicolumn{2}{|c|}{ Clinician Suspicion } \\
\hline & $\begin{array}{c}<1 \% \\
(N=9,252)\end{array}$ & $\geq 1 \%(N=2,667)$ \\
\hline Death due to the intra-abdominal injury & $1(0.01) 0-0.1$ & $8(0.3) 0.1-0.6$ \\
\hline Therapeutic intervention at laparotomy & $16(0.2) 0.1-0.3$ & 98 (3.7) $3.0-4.5$ \\
\hline Angiographic embolization due to bleeding from the intra-abdominal injury & $2(0.02) 0-0.1$ & 9 (0.3) $0.2-0.6$ \\
\hline Blood transfusion for anemia secondary to intra-abdominal hemorrhage & $16(0.2) 0.1-0.3$ & $106(4.0) 3.3-4.8$ \\
\hline $\begin{array}{l}\text { Administration of intravenous fluids for at least two nights in patients with pancreatic or } \\
\text { gastrointestinal injuries }\end{array}$ & $12(0.1) 0.1-0.2$ & $67(2.5) 2.0-3.2$ \\
\hline
\end{tabular}

Table 5

Indications Cited for Obtaining Abdominal CT Scans When Clinician Suspicion for Intra-abdominal Injury Undergoing Acute Intervention Was Very Low $(<1 \%)$

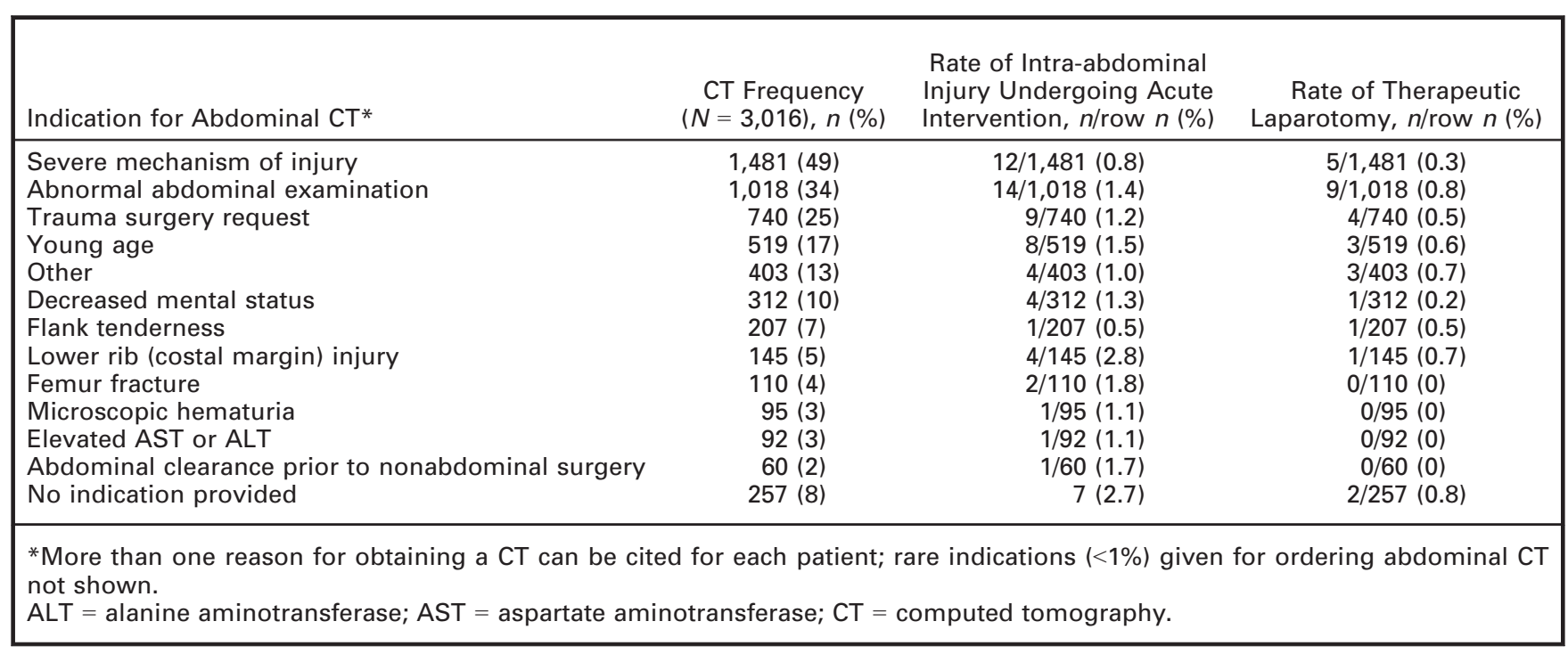

Indiscriminate use of CT is not without risks as it leads to increased health care costs, increased risk of contrastinduced nephropathy, and most importantly, increased risk of radiation-induced malignancies. The risk of a solid organ cancer was recently estimated to occur in one out of every 300 to 390 girls undergoing abdominal CT and one out of every 670 to 760 boys undergoing abdominal CT. 8

Clinical prediction rules are decision support tools that use clinical findings (history, physical examination, and/or simple screening test results) to assist clinicians in patient evaluation and care. When appropriately applied, they can change clinical behavior and reduce unnecessary costs while maintaining quality of care and patient satisfaction. ${ }^{16,17}$ In the parent study, a prediction rule that uses routinely obtained patient history and physical examination findings was derived to stratify children with blunt torso trauma into different risk groups for intra-abdominal injuries undergoing acute intervention. The current analysis reveals that the derived prediction rule had substantially greater sensitivity than unstructured clinician suspicion in correctly identifying these children. Unstructured clinician suspicion of $<1 \%$ would have missed nearly $20 \%$ of children with intra-abdominal injuries undergoing acute intervention. Only $3 \%$ of patients with intra-abdominal injuries undergoing acute intervention, however, were not identified by the clinical prediction rule. Three of these patients had clinician suspicion $<1 \%$ for intraabdominal injury undergoing acute intervention, so were also considered very low risk by clinician suspicion. The other three children undergoing intervention had higher clinician suspicions.

Of note, unaided clinician suspicion for intra-abdominal injury undergoing acute intervention had higher specificity than the prediction rule. Nonetheless, ED physicians often did not practice in accordance with their clinical suspicion, as they obtained abdominal CT scans in nearly one-third of children in whom their suspicion $<1 \%$. 
In the parent study, $25 \%$ of patients considered very low risk by the prediction rule underwent an abdominal CT scan. This represents $23 \%$ of all abdominal CT scans obtained, suggesting that a substantial number of unnecessary CT scans can be obviated with the use of the prediction rule. ${ }^{18}$ The high NPV and improved LRof the prediction rule could help decrease unnecessary abdominal CT scans in children without missing those patients who require acute intervention. In contrast, deciding not to image with CT based solely on a clinician suspicion of $<1 \%$ would miss more cases of intraabdominal injuries undergoing acute intervention than application of the prediction rule. If validated, this prediction rule could decrease the variability in ordering CT scans for children with blunt torso trauma and thus enhance the quality of care. Once validated, translation of this rule into practice will be necessary. Knowledge translation for implementation of pediatric traumatic brain injury prediction rules is currently being studied, ${ }^{25}$ and application of the abdominal CT rule could follow similar methods.

We identified the reasons given by clinicians for obtaining abdominal CT scans when they believed patients to be at very low risk. Most of these reasons were of very low yield and uncommonly resulted in identifying patients undergoing specific therapy. The top three reasons provided for obtaining CT scans despite $<1 \%$ clinician suspicion were severity of mechanism of injury, abnormal abdominal examination, and trauma team request. Although one of these indications for obtaining CT scans in spite of the low clinician suspicion is a variable in the derived prediction rule (abnormal abdominal examination), the other two are not. The combination of very low clinician suspicion and the absence of all the prediction rule variables has a potential to identify a subset of patients who are at very low risk for intra-abdominal injury undergoing acute intervention and in whom abdominal CT scan is very unlikely to be helpful.

Two prior studies evaluated clinicians' predictive abilities for identifying adult patients with intra-abdominal injuries. In one study, physicians recorded their impressions of intra-abdominal injury ( $>50,>10$ to $50,>5$ to 10 , 1 to 5 , or $<1 \%$ ). ${ }^{15}$ In the second study, physicians ranked their clinical impressions into five ordinal categories of likelihood of an intra-abdominal injury, from "most likely" to "no suspicion," taking into consideration the clinical examination, basic laboratory results, plain radiography, and the FAST examination. ${ }^{26}$ Both studies revealed an appropriately increasing LR (range $=11$ to 19) for intra-abdominal injury as clinician's impression of the probability of an injury increased. Similar to our study, both of these studies reported cases of intra-abdominal injuries in patients with the lowest clinician suspicion. They also reported frequent use of abdominal CT in patients considered by the clinicians to be at lowest risk of intra-abdominal injury. Unfortunately, we could find no comparable studies in the pediatric literature.

Clinical prediction rules are tools to improve clinical decision-making, but few have undergone rigorous impact analysis to determine their actual effects on patient care. The traditional research model for predic- tion rules includes derivation of the rule followed by validation and impact analysis. ${ }^{16,17,27}$ Some investigators recommend measuring unstructured clinician decisionmaking in the derivation and validation phases of clinical prediction rules because it is possible that the clinicians' estimates may be superior or at least equivalent to the prediction rule, thus minimizing the importance of the rule. ${ }^{27}$ Here, we report the comparison of unstructured clinician suspicion with the prediction rule and believe that these results can enhance physician confidence in evaluating children with blunt torso trauma using the clinical prediction rule.

\section{LIMITATIONS}

The study sites include a substantial number of tertiary care pediatric EDs where rates of CT use are likely lower than those in nonchildren's hospitals or general EDs. ${ }^{28}$ Furthermore, the actual performance of clinician suspicion and CT ordering practices between providers of different specialty training is unknown. Studies on cranial CT use in children with blunt head trauma suggested different ordering patterns between pediatricians, emergency providers, and pediatric emergency-trained physicians. ${ }^{12,28}$ Clinician decision-making and abdominal CT ordering patterns need similar study. Furthermore, the clinical prediction rule was specifically designed to identify all patients with injuries undergoing acute intervention, and therefore specificity was sacrificed for sensitivity. ${ }^{18}$ We intentionally chose to exclude patients with intra-abdominal injuries not undergoing specific intervention, to reduce misclassification bias that would occur due to false-positive CT scans. For example, in a study of 5,000 patients undergoing CT scans, we would expect, assuming a specificity of $99 \%$, that approximately 50 patients would be misclassified as having intra-abdominal injuries by CT scan. Furthermore, some patients with solid organ injuries may be hospitalized for observation either based on practice pattern variation or as an institutional practice, thus affecting the test characteristics of the derived prediction rule. In addition, the clinical prediction rule was modeled on a clinical outcome and therefore it would be methodologically inappropriate to compare clinician suspicion for a radiographic outcome to a prediction rule for a clinical outcome.

In addition, CT scans were not obtained on all patients, so some radiographically present but clinically silent injuries may have been missed. However, followup was performed on all patients to identify those with our patient-oriented outcome. Finally, clinicians were not specifically asked how their suspicion affected CT decision-making, and very low suspicion did not equate to not obtaining an abdominal CT.

\section{CONCLUSIONS}

A clinical prediction rule had a significantly higher sensitivity than clinician suspicion for identifying intraabdominal injury undergoing acute intervention, but a lower specificity. The higher specificity of clinician suspicion, however, did not translate into clinical practice, as clinicians frequently obtained abdominal computed tomography scans in patients they considered to be at 
very low risk. If validated, this clinical prediction rule can assist in clinical decision-making around computed tomography use after blunt abdominal trauma in children by limiting computed tomography scan use in lowrisk patients.

We acknowledge the efforts of the following individuals participating in PECARN at the time this study was initiated.

PECARN Steering Committee: N. Kuppermann, Chair; E. Alpern, D. Borgialli, J. Callahan, J. Chamberlain, P. Dayan, J. M. Dean, M. Gerardi, M. Gorelick, J. Hoyle, E. Jacobs, D. Jaffe, R. Lichenstein, K. Lillis, P. Mahajan, R. Maio, F. Moler, D. Monroe, R. Ruddy, R. Stanley, M. Tunik, A. Walker.

MCHB/EMSC liaisons: D. Kavanaugh, H. Park.

PECARN Data Coordinating Center: M. Dean, R. Holubkov, S. Knight, A. Donaldson, S. Zuspan, M. Miskin, J. Wade, A. Jones, M. Fjelstad.

Feasibility and Budget Subcommittee: T. Singh, Chair; A. Drongowski, L. Fukushima, E. Kim, D. Monroe, G. O'Gara, H. Rincon, M. Tunik, S. Zuspan.

Grants and Publications Subcommittee: M. Gorelick, Chair; E. Alpern, D. Borgialli, K. Brown, L. Cimpello, A. Donaldson, G. Foltin, F. Moler, S. Teach.

Protocol Concept Review and Development Subcommittee: D. Jaffe, Chair; J. Chamberlain, A. Cooper, P. Dayan, J. M. Dean, R. Holubkov, P. Mahajan, R. Maio, N. C. Mann, K. Shaw, A. Walker.

Quality Assurance Subcommittee: R. Stanley, Chair; P. Ehrlich, R Enriquez, M. Gerardi, R. Holubkov, E. Jacobs, R. Lichenstein, K Lillis, B. Millar, R. Ruddy, M. Shults.

Safety and Regulatory Affairs Subcommittee (SRAS): W. Schalick, J. Callahan, Co-Chairs; S. Atabaki, J. Burr, K. Call, J. Hoyle, R. Ruddy, J. Suhajda, N. Schamban.

We thank the research coordinators in PECARN, without whose dedication and hard work this study would not have been possible; and all the clinicians around the PECARN who enrolled children in this study.

\section{References}

1. Heron M. Deaths: leading causes for 2007. Natl Vital Stat Rep 2011;59:1-95.

2. Shuman WP, Ralls PW, Balfe DM, et al. Imaging of blunt abdominal trauma: American College of Radiology: ACR appropriateness criteria. Radiology 2000;215(Suppl):143-51.

3. Broder J, Fordham LA, Warshauer DM. Increasing utilization of computed tomography in the pediatric emergency department, 2000-2006. Emerg Radiol 2007;14:227-32.

4. Broder J, Warshauer DM. Increasing utilization of computed tomography in the adult emergency department, 2000-2005. Emerg Radiol 2006;13: 25-30.

5. Brenner DJ, Hall EJ. Computed tomography-an increasing source of radiation exposure. N Engl J Med 2007;357:2277-84.

6. Frush DP, Frush KS. The ALARA concept in pediatric imaging: building bridges between radiology and emergency medicine: consensus conference on imaging safety and quality for children in the emergency setting, Feb. 23-24, 2008, Orlando, FL - Executive Summary. Pediatr Radiol 2008;38(Suppl 4): S629-32.

7. Committee to Assess Health Risks from Exposure to Low Levels of Ionizing Radiation NRC. Health Risks from Exposure to Low Levels of Ionizing Radiation: BEIR VII, Phase 2. Washington, DC: The National Academies Press, 2006.

8. Miglioretti DL, Johnson E, Williams A, et al. The use of computed tomography in pediatrics and the associated radiation exposure and estimated cancer risk. JAMA Pediatr 2013;167:700-7.

9. Mathews JD, Forsythe AV, Brady Z, et al. Cancer risk in 680,000 people exposed to computed tomography scans in childhood or adolescence: data linkage study of 11 million Australians. BMJ. 2013; 346: f2360.

10. Pearce MS, Salotti JA, Little MP, et al. Radiation exposure from CT scans in childhood and subsequent risk of leukaemia and brain tumours: a retrospective cohort study. Lancet 2012;380:499 505.

11. Larson DB, Johnson LW, Schnell BM, Goske MJ, Salisbury SR, Forman HP. Rising use of CT in child visits to the emergency department in the United States, 1995-2008. Radiology 2011;259:793-801.

12. Blackwell CD, Gorelick M, Holmes JF, Bandyopadhyay S, Kuppermann N. Pediatric head trauma: changes in use of computed tomography in emergency departments in the United States over time. Ann Emerg Med 2007;49:320-4.

13. Palchak MJ, Holmes JF, Kuppermann N. Clinician judgment versus a decision rule for identifying children at risk of traumatic brain injury on computed tomography after blunt head trauma. Pediatr Emerg Care 2009;25:61-5.

14. Bandiera G, Stiell IG, Wells GA, et al. Canadian C-Spine and CT Head Study Group. The Canadian C-spine rule performs better than unstructured physician judgment. Ann Emerg Med 2003;42:395402.

15. Holmes JF, Wisner DH, McGahan JP, Mower WR, Kuppermann N. Clinical prediction rules for identifying adults at very low risk for intra-abdominal injuries after blunt trauma. Ann Emerg Med 2009;54:575-84.

16. Wasson JH, Sox HC, Neff RK, Goldman L. Clinical prediction rules. Applications and methodological standards. N Engl J Med 1985;313:793-9.

17. Laupacis A, Sekar N, Stiell IG. Clinical prediction rules. A review and suggested modifications of methodological standards. JAMA 1997;277:488-94.

18. Holmes JF, Lillis K, Monroe D, et al. Pediatric Emergency Care Applied Research Network (PECARN). Identifying children at low risk of clinically important blunt abdominal trauma. Ann Emerg Med 2013;62:107-16.

19. Jindal A, Velmahos GC, Rofougaran R. Computed tomography for evaluation of mild to moderate pediatric trauma: are we overusing it? World J Surg 2002;26:13-6. 
20. Awasthi S, Mao A, Wooton-Gorges SL, Wisner DH, Kuppermann N, Holmes JFJr. Is hospital admission and observation required after a normal abdominal computed tomography scan in children with blunt abdominal trauma? Acad Emerg Med 2008;15:895-9.

21. Ruess L, Sivit CJ, Eichelberger MR, Gotschall CS, Taylor GA. Blunt abdominal trauma in children: impact of CT on operative and nonoperative management. AJR Am J Roentgenol 1997;169:1011-4.

22. Holmes JF, London KL, Brant WE, Kuppermann N. Isolated intraperitoneal fluid on abdominal computed tomography in children with blunt trauma. Acad Emerg Med 2000;7:335-41.

23. Berdahl CT, Vermeulen MJ, Larson DB, Schull MJ. Emergency department computed tomography utilization in the United States and Canada. Ann Emerg Med 2013;62:486-94.

24. Kocher KE, Meurer WJ, Fazel R, Scott PA, Krumholz HM, Nallamothu BK. National trends in use of computed tomography in the emergency department. Ann Emerg Med 2011;58:452-62.

25. Sheehan B, Nigrovic LE, Dayan PS, et al. Pediatric Emergency Care Applied Research Network (PECARN). Informing the design of clinical decision support services for evaluation of children with minor blunt head trauma in the emergency department: a sociotechnical analysis. J Biomed Inform 2013;46:905-13.

26. Poletti PA, Mirvis SE, Shanmuganathan $K$, et al. Blunt abdominal trauma patients: can organ injury be excluded without performing computed tomography? J Trauma 2004;57:1072-81.
27. Schriger DL, Newman DH. Medical decision making: let's not forget the physician. Ann Emerg Med 2012;59:219-20.

28. Stanley RM, Hoyle JD Jr, Dayan PS, et al. Pediatric Emergency Care Applied Research Network (PECARN). Emergency department practice variation in computed tomography use for children with minor blunt head trauma. J Pediatr 2014; 165:1201-6.

\section{APPENDIX A}

Participating centers and site investigators are listed below in alphabetical order: Bellevue Hospital Center (M. Tunik); Children's Hospital Boston (L. Lee); Children's Hospital of Michigan (P. Mahajan); Children's Hospital of Philadelphia (A. Ellison); Children's Hospital of Wisconsin (K. Yen); Children's National Medical Center (S. Atabaki); Cincinnati Children's Hospital Medical Center (B. Kerrey); DeVos Children's Hospital (J. Kooistra); Howard County Medical Center (D. Monroe); Hurley Medical Center (D. Borgialli); Jacobi Medical Center (S. Blumberg); Nationwide Children's Hospital (B. Bonsu); New York Presbyterian-Morgan Stanley Children's Hospital (M. Kwok); Primary Children's Medical Center (K. Adelgais); St. Louis Children's Hospital (K. Quayle); University of California Davis Medical Center (J. Holmes, N. Kuppermann); University of Maryland (J. Menaker); University of Michigan (A. Rogers); University of Rochester (M. Garcia); Women and Children's Hospital of Buffalo (K. Lillis).

\section{VIRTUAL ISSUES}

"Virtual Issues" are now a key feature of Academic Emergency Medicine's home page. A virtual issue is basically just a collection of articles on a given topic. The idea is that a reader will go there to look for a particular issue, but then will see our other offerings on that topic, as well, increasing our full-text download numbers and helping insure the broadest dissemination of our authors' work.

We now have five "virtual issues" online. Go to the journal's home page on the Wiley Online Library (WOL) platform - "Find Issues" on the left-hand side and click on the feature. In addition to the initial geriatrics one, the following are up and running on: ultrasound, toxicology, injury prevention, statistics and research methodology. Again, consult the "Find Issues" area and click on the desired issue. They can also be accessed from the "Virtual Issues" module, right column, on the journal's home page.

http:/onlinelibrary.wiley.com/journal/10.1111(ISSN)1553-2712.

STAY TUNED FOR UPDATES AS MORE VIRTUAL ISSUES ARE ADDED. IF YOU WOULD LIKE TO COMPOSE ONE, PLS CONTACT DAVE CONE, MD, Or SANDI ARJONA. 